\title{
Fabrication-error analysis of injection-molded aspheric elements using typical aberration terms in transmitted wavefront with Shack-Hartmann wavefront-sensing measurement
}

\author{
Xuemin Cheng $\odot,{ }^{\mathrm{a}, *, \dagger}$ Lei Yan, ${ }^{\mathrm{b}, \dagger}$ Lingcheng Liu, ${ }^{\mathrm{a}}$ Jie Cao $\odot,{ }^{\mathrm{b}}$ \\ Yu Jen Lin, ${ }^{\mathrm{c}}$ and Qun Hao ${ }^{\mathrm{b}, *}$ \\ ${ }^{a}$ Tsinghua University, Shenzhen International Graduate School, Shenzhen, China \\ ${ }^{b}$ Beijing Institute of Technology, School of Optics and Photonics, Beijing, China \\ ${ }^{\mathrm{c}}$ Genius Electronic Optical Co., Ltd., Xiamen, China
}

\begin{abstract}
Aspheric lenses help meet the most demanding optical requirements while the precision injection molding technique hits the target for precision and cost. We developed a method of analyzing aberration terms in the transmitted "wavefront measurement," determined by Shack-Hartmann wavefront sensing to estimate the fabrication errors of injection-molded aspheric elements. Considering aspheric element fabrication using a small training data set and $F$-measure fuzzy cluster analysis, an unsupervised learning method was applied to extract typical aberration terms from the wavefront polynomial. The experimental results suggest that these aberration terms, which are related to spherical (third-, fifth-, and seventh-order) and coma (third-order) aberration terms in the transmitted wavefront polynomial expansion, can be employed to estimate the surface error and decenter, respectively, of a lens from a specific mold cavity. The sampling lenses evaluated in the proposed measuring process were collected from different mold cavities according to their total working performance in the modulated transfer function measurement for the whole camera module. The performances of the typical aberration terms were discussed by comparing to the ones obtained from an interferometer and a profilometer. The proposed method could provide high detection efficiency and can thus be applied for the quality control of aspheric elements for mobile phones, where the existing errors are mainly spherical, coma, and astigmatism aberrations. (C) The Authors. Published by SPIE under a Creative Commons Attribution 4.0 Unported License. Distribution or reproduction of this work in whole or in part requires full attribution of the original publication, including its DOI. [DOI: 10.1117/1.OE.59.12.123102]
\end{abstract}

Keywords: fabrication-error analysis; Shack-Hartmann wavefront sensing; fuzzy cluster analysis; typical aberration terms; aspheric elements.

Paper 20201039 received Sep. 2, 2020; accepted for publication Nov. 30, 2020; published online Dec. 12, 2020.

\section{Introduction}

Injection-molded aspheric lenses have widespread applications in industrial and consumer electronics, ${ }^{1,2}$ among other fields. However, during injection molding, surface errors/deformations, surface decentering and asymmetry, element internal stress birefringence, and nonuniform refractive indices are almost inevitable. ${ }^{3}$ Measurements are required to identify these errors, optimize the injection-molding characteristics to compensate for them, and modify the prototype molding parameters. ${ }^{4-7}$ Currently, aspheric element measurements are mainly achieved by either contact or noncontact approaches to estimate the aspheric surface error. ${ }^{8}$ Due to the single-sided physical geometry of the measured injection-molded lens, the imaging quality cannot be determined directly. Therefore, the transmitted wavefront measurement method, which provides more comprehensive information for error estimation in injection molding, is used to adjust the processes to achieve error compensation.

*Address all correspondence to Xuemin Cheng, chengxm@sz.tsinghua.edu.cn; Qun Hao, qhao@bit.edu.cn

${ }^{\dagger}$ These authors contributed equally to this work. 
Of all transmitted wavefront measurement methods, the most commonly employed approaches focus on evaluating interference patterns and employing the Shack-Hartmann (SH) method. Meanwhile, interferometry can provide extremely high accuracy, although it requires either additional correction elements for compensation or stitching interferometry to reduce the difficulty of interference fringe analysis. ${ }^{9}$ Moreover, these two interferometry techniques have limited dynamic test ranges and protracted testing periods. ${ }^{10-13}$ Shack-Hartmann wavefront sensing (SHWFS) can be utilized to reconstruct the wavefront directly from the slope obtained in a single trial. ${ }^{14,15}$ Therefore, researchers have investigated SHWFS measurements and the relationships between fabrication errors and SHWFS-measured transmitted wavefronts.

In 2008, Lai et al. ${ }^{16}$ analyzed injection-molding conditions using numerical simulations. They showed that the residual birefringence is generated in the mold-filling stage and that the third-order spherical aberration (SA) is sensitive to the injection-molding process conditions. $\mathrm{Li}$ et al. used finite element analysis to simulate the injection-molding process conditions. The results obtained indicated that a predefined inhomogeneous refractive index and injectionmolding-induced warpage led to a distorted transmitted wavefront. ${ }^{17}$ Moreover, Huang et al. ${ }^{18}$ conducted numerical simulations of the residual birefringence of injection-molded lenses. Combined with SHWFS experiments, these simulations showed that the transmitted wavefront was affected by refractive index changes and residual birefringence errors in injection-molded aspheric lenses. Overall, these studies clearly demonstrated that the transmitted wavefront can be employed as a lens quality indicator. Thus, developing a method to extract typical aberration terms from a complex wavefront and evaluate the lens quality has become a crucial problem in wavefront measurement.

Learning-based methods ${ }^{19}$ have been used to solve inverse problems in quantifying optical image distortions. The technique shows that it requires several hours or even days to collect tens of thousands of labeled data experimentally for neural network training. This approach is not readily feasible, particularly when applying error estimates of defective injection-molded aspheric elements. To solve this problem, this report employs $F$-measure fuzzy cluster methods (F-FCMs) to extract typical aberration terms. Accordingly, a small training data set (opticalcomponent structured error types for different molds under stable fabrication of an aspheric lens in a mold cavity and stable measurement conditions and their corresponding lenses) can be applied in practice. The unsupervised learning method used in FCMs is combined with the physical fabrication procedure of precision injection molding. Moreover, following the extraction of typical aberration terms by F-FCM analysis, typical experimental results were evaluated in comparison with interferometer and profilometer results. Thus, the simulation and experimental results verify the validity of the proposed error evaluation methods.

The remainder of this paper is structured as follows. Section 2 discusses the proposed methods, including those used for problem analysis, model building, and solution-finding. Section 3 describes the extraction of typical wavefront aberration terms. Section 4 presents an experimental analysis for the classification of mold cavities with different surface errors using a profilometer, an interferometer, and SHWFS. Finally, Sec. 5 summarizes the primary conclusions of this study and future research directions.

\section{Methods}

\subsection{Transmitted Wavefront with Shack-Hartmann Wavefront-Sensing Measurement}

Figure 1 shows the experimental setup of the wavefront-sensing technique. The light wave propagates through a fiber, a collimating lens, and a focusing lens, creating a fixed ideal point source and transmitting through the effective aperture of the tested lens. Then the collimated light is expanded to fully exploit the effective area of the wavefront sensor. The measured transmitted wavefront, ${ }^{20} W(\rho, \theta)$, can be expressed using a Zernike polynomial expansion (a detailed representation is provided in Appendix A). The Zernike representation of the wavefront aberration function is given by Eq. (1) with 36 terms. $^{21}$ 


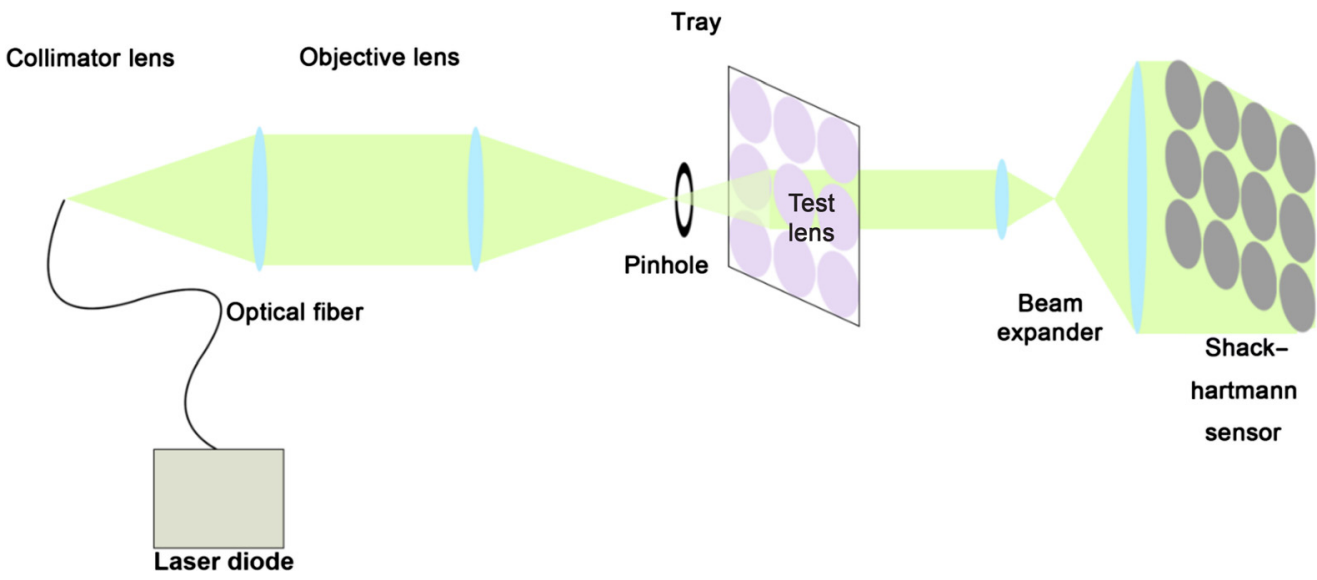

Fig. 1 Schematic diagram of experimental setup.

$$
W(\rho, \theta)=\sum_{k=1}^{36} Z_{k} C_{k}(\rho, \theta)
$$

where $\rho_{i}$ is the polar coordinate system radius, $\theta_{i}$ denotes the angle measured from the polar axis.

The wavefront measurement absolute accuracy root-mean-square (RMS) of the wavefront testing system is $1 / 20 \lambda$, and its wavefront-sensing validity and technical parameters are shown in Appendix C.

\subsection{Materials}

The tested aspheric lens is shown in Fig. 2(a). Its two sides are denoted as A1 and A2 and the effective aperture of the lens is $1 \mathrm{~mm}$ in diameter, which is used as the measuring area for evaluation and comparison. This lens is used as the stop aperture in a mobile-phone camera lens, which takes the layout similar to that of the patented lens in Fig. 2(b). The aspheric lens discussed was an optical element formed from a high-molecular polymer and fabricated by injection molding. ${ }^{22}$ As a stable and precise large-scale fabrication process, its geometric fabrication

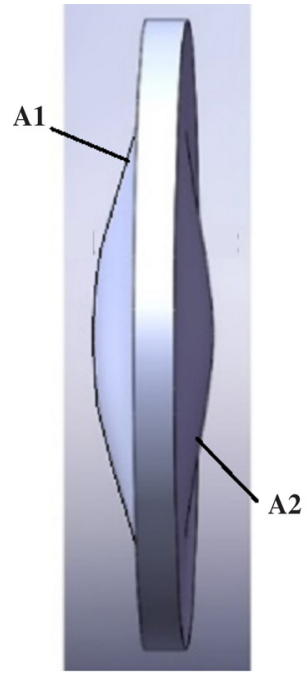

(a)

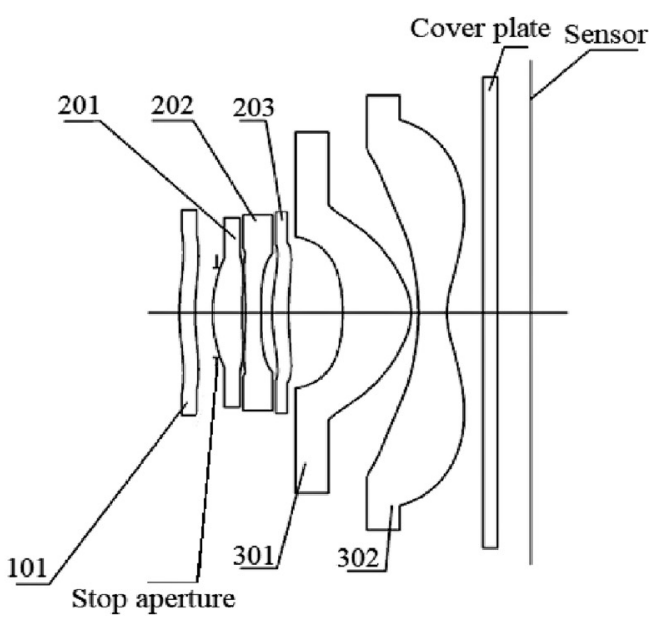

(b)

Fig. 2 (a) Aspherical surface of experimental sample lens (surfaces A1 and A2 are both rotationally symmetric-aspherical, with an external diameter of $1.2 \mathrm{~mm}$, effective optical aperture of $1 \mathrm{~mm}$, and lens thickness of $0.42 \mathrm{~mm}$ ). (b) Mobile-phone camera module (US20160195698A1). 


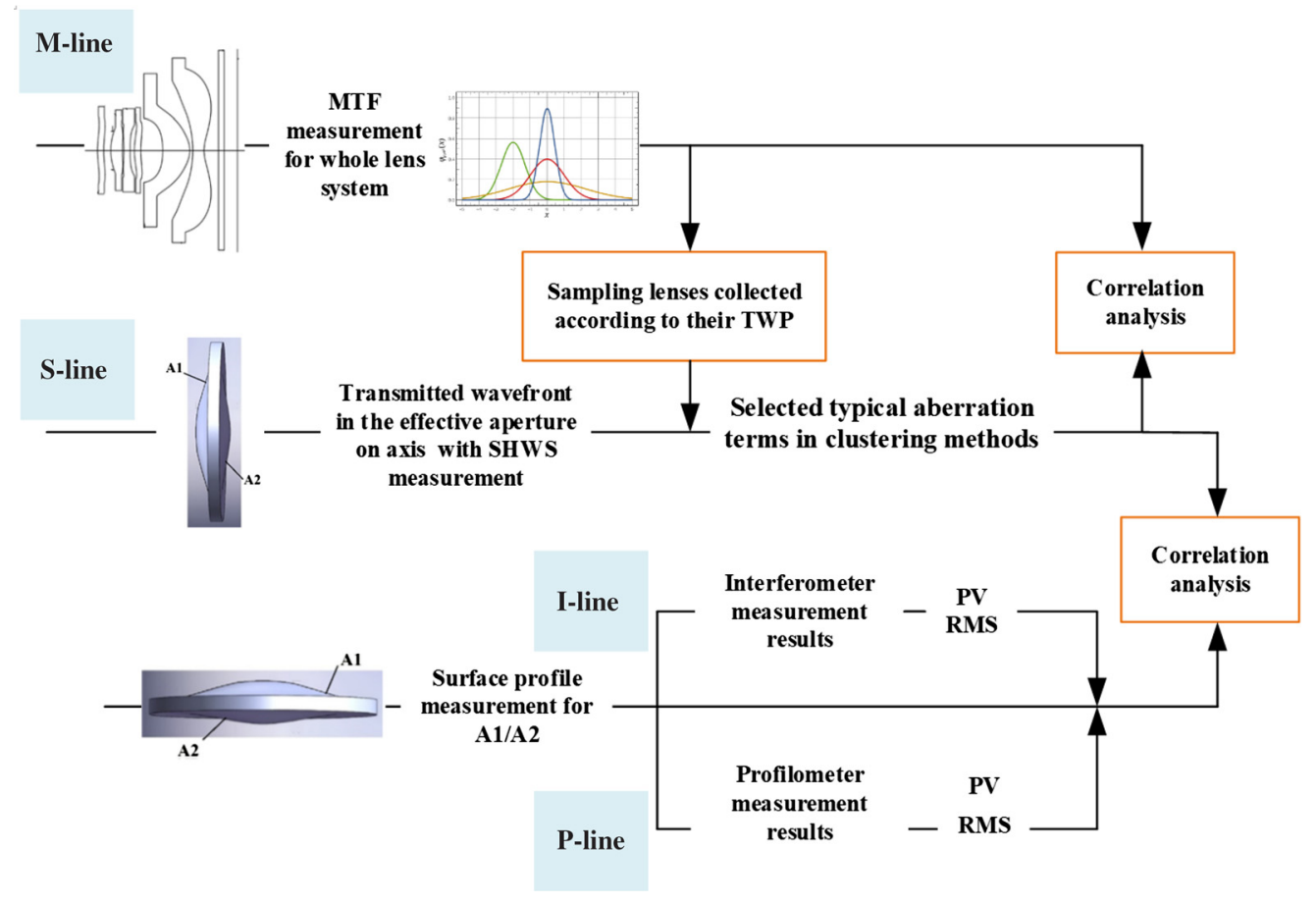

Fig. 3 Schematic diagram of lens measurements and evaluation procedure using the I-line, P-line, M-line, and the proposed S-line in the production line.

accuracy was measured, and the fabrication parameters of the mold cavity were monitored and adjusted to achieve superior optical performance. ${ }^{23}$ Figure 3 shows the measuring schemes: measuring the working performance of the integrated camera module and measuring the single optical element.

In Fig. 3, M-line is for the modulated transfer function (MTF) measurement for whole camera module; in this way, the total working performance (TWP) of one mold cavity was evaluated as follows: hundreds of tested lenses from one mold cavity were integrated into their whole camera module [as shown in Fig. 2(b)], and then their lens performances could be evaluated in the whole lens system; then, the system imaging quality, mainly indicated by their MTF values, can be quantified according to the testing standard of the camera module; therefore, the TWP for different mold cavities was evaluated. The TWP of a mold cavity was assigned the label no good (NG) part when most tested lenses consistently failed to achieve a reliable quality upon integration. The label trial group was assigned when the tested lenses could not achieve a stable performance, and the fabrication parameters needed adjusted and optimized. In this way, the TWP describes that the fabrication ability of the mold cavity system, whereas the percentage, e.g., 93\%, of the tested lenses revealed a reliable mold cavity possessing satisfactory system imaging quality.

In Fig. 3, I-line and P-line are employed for surface profile measurement of sides A1 and A2 of the lens element using the profilometer and the interferometer described in Appendix B, respectively. These qualities are evaluated using peak-to-valley (PV) and RMS values for surface errors, and using the deviation of the conic part for foci location error.

In our study, S-line is used to measure the transmitted wavefront of the lens element with SHWS method, in which their typical aberration terms or the term combinations (as shown in Fig. 3) are discussed and analyzed so that the evaluation of fabrication ability of the mold cavity system could be realized. As we all know, the aberration functions for wavefront have been investigated, e.g., Zernike polynomials, the polynomial representation of wavefront aberration has been developed up to the fifth-order in the previous research work, ${ }^{24}$ and the polynomial has a definite expression corresponding to the optical performance..$^{25,26}$

These three measuring lines (M-line, I-line, and P-line) were employed, and their effectiveness has been proved in the production line of the lens element; thus, their results are used together to view the correlation analysis for selected typical aberration terms in transmitted 
wavefront. First, in consideration of classification for small-scale datasets, the sampling lenses in the proposed S-line were collected from different mold cavities according to their TWP in the M-line; then it is ensured that the errors were distributed as diversely as possible (inter-cluster) with different percentage reliability values or regarded as experimental trial or NG samples; the overall repeatability of injection molding, generally $<0.7 \%$ to assure the stable fabrication of an aspheric lens in a mold cavity, ${ }^{27}$ ensures a stable consistency in the wavefront aberration (intra-cluster). In this way, the typical wavefront aberration terms are selected using the fuzzy clustering method analyzed in Sec. 2.3; further, these terms were characterized in the evaluation experiments when analyzing their associations with the I-line and P-line measuring results.

\subsection{Fuzzy Clustering Method}

Clustering methods can be roughly divided into two groups, namely, hierarchical and partitional methods. ${ }^{28}$ The results of hierarchical methods are represented as dendrograms, where each branch represents a group of samples with similar characteristics; however, hierarchical methods suffer from the non-uniqueness of the dendrogram. ${ }^{29}$ The objective of partitional methods is to find the best partition of samples for clusters, in such a way that one criterion (e.g., the total inertia of the clusters) is optimized. ${ }^{30}$ When the objective function assumes the smallest value, each sample in the same group exhibits the smallest difference, and the differences among distinct groups became the largest. Meanwhile, because the classification only subdivided the data, it did not necessarily have a physical meaning. ${ }^{31}$ Therefore, the FCM algorithm could be applied, and the $F$-measure parameter $(F$, the weighted average of the $F$-measures of all the mold cavities) was introduced to describe the validity of the classification. In this study, the sensitive aberration terms with better cluster structures can also yield reliable data in geometric structures. Based on the above choice, F-FCM can be applied more efficiently.

The fuzzy clustering algorithm links each lens to a cluster index via a real-valued vector. The measured lenses were defined as the initial sample data set $X$, namely, $\mathrm{X}=\left\{\chi_{1}, \chi_{2} \cdots \chi_{n}\right\} \subset R^{s}, \quad \chi_{k}=\left\{\chi_{k 1}, \chi_{k 2} \cdots, \chi_{k M}\right\}^{\mathrm{T}} \subset R^{s}$, where $\chi_{k}$ corresponds to the Zernike term of each lens. The index vector $J(U, P)$ defines the lens membership with respect to various clusters:

$$
\begin{gathered}
J(U, P)=\sum_{k=1}^{c} \sum_{i=1}^{\mathrm{n}} u_{k i}^{m} d_{k i}^{2}, \quad m \in[1, \infty] \quad \text { s.t } U \in M_{f c}, \\
d_{k i}^{2}=\left\|\chi_{i}-P_{k}\right\|=\left(\chi_{i}-P_{k}\right)^{T} A_{k}\left(\chi_{i}-P_{k}\right), \\
M_{f c}=\left\{U \in R^{c n} \mid u_{k i} \in[0,1] \forall k \sum_{i=1}^{c} u_{k i}=1 ; \forall i 0<\sum_{i=1}^{c} u_{k i}<n\right\},
\end{gathered}
$$

where $1 \leq i \leq n$ and $1 \leq k \leq c$. In Eq. (2), $c$ and $n$ are the numbers of clusters and samples (or lenses) in the data set, respectively; $m$ is a real-valued number that controls the fuzziness of the resulting clusters; $u_{k i}$ is the degree of membership of lens $x_{i}$ in cluster $k$; and $d_{k i}^{2}$ denotes the square of the distance from lens $\chi_{i}$ to centroid $P_{k}$. Moreover, $m$ is any real-valued number greater than one ( $m=2$ in the experiments). $u_{k i}$ and $P_{k}(k=1,2 \cdots c)$ can be obtained after minimizing the total inertia criterion. ${ }^{32}$ Meanwhile, the values of $u_{k i}$ lie between 0 and 1 for the vector components. For a given lens, an index close to one indicates a strong association with the cluster. Conversely, index values close to zero indicate the absence of a strong association with the corresponding cluster. In Eq. (3), $A_{k}$ is a symmetric and positive definite matrix. When $A_{k}$ is the identity matrix, $d_{k i}^{2}$ corresponds to the square of the Euclidian distance. Equation (4) indicates that empty clusters are not allowed. Further, it shows that the parameters of interest are $P_{k}$ and $u_{k i}$. These parameters can be obtained in the four steps. ${ }^{33}$

1. Initialize: Fix $c$ and $m$ and calculate $d_{k i}^{2}$. Experimentally, $c$ is equal to the number of chosen cavities. Select $c$ samples as initial centroids $P_{k}^{0}$, and then form partitions of all other samples around these centroids to obtain the initial partition matrix, $U_{0}=\left[u_{k i}\right]$, $k=1, \ldots, c$ and $i=1, \ldots, n$. At step 1, perform the following procedure. 
2. Compute centroid $P_{k}^{l}$ :

$$
P_{k}^{l}=\frac{\sum_{i=1}^{n} u_{k i}^{(l-1) m} \chi_{i}}{\sum_{i=1}^{n} u_{k i}^{(l-1) m}}
$$

3. Compute membership values $u_{k i}^{l}$ :

$$
u_{k i}^{l}=\left\{\begin{array}{ccc}
\frac{1}{\sum_{j}^{c}\left(\frac{d_{k i}^{l}}{d_{k i}^{l}}\right)^{\frac{2}{m-1}}}, & I_{i} \neq \oslash ; & I_{i}=\left\{k \mid 1 \leq k \leq c ; d_{k i}^{2}=0\right\}, \\
0, \forall i \in \tilde{I}_{i} ; \frac{1}{\left|I_{i}\right|}, & \forall i \in I_{i} & \tilde{I}_{i}=\{1,2, \cdots c\}-I_{i} .
\end{array}\right.
$$

4. Repeat procedures (b) and (c) until the objective function is minimized and stabilized, i.e., $\left\|J^{l}-J^{l-1}\right\| \leq \varepsilon, l>1$, where $\varepsilon$ denotes the error between two consecutive values of the constrained objective function.

After several rounds through Eqs. (5) and (6), the algorithm stops when the error between two consecutive values of the constrained fuzzy partition matrix, $U$, is smaller than an a priori specified value. The typical Zernike terms are expected to exhibit a clustering effect, because the types of lens fabrication errors from different mold cavities follow a specific distribution. For lenses from the same mold cavity, the typical Zernike terms should be clustered closely together; therefore, an evaluation function for the clustering effect was defined using the silhouette merit function proposed by Rousseeuw. ${ }^{32}$ The silhouette $s\left(\chi_{i}\right)$ of the Zernike terms is given by the ratio in Eq. (7):

$$
s\left(\chi_{i}\right)=\frac{b\left(\chi_{i}\right)-a\left(\chi_{i}\right)}{\max \left\{a\left(\chi_{i}\right), b\left(\chi_{i}\right)\right\}},
$$

where $t_{r}$ is the cluster to which $\chi_{i}$ belongs, $a\left(\chi_{i}\right)$ is defined as the average distance between $\chi_{i}$ and all other points in $t_{r}$. For any other cluster, $t_{s} \neq t_{r}$, and let $d\left(x_{i}, t_{s}\right)$ denote the average distance from $x_{i}$ to all points of $t_{s}$. Furthermore, $b\left(\chi_{i}\right)$ is the smallest of $d\left(\chi_{i}, t_{s}\right), r \neq s=1, \ldots, c$. The silhouette value lies between -1 and 1 . When its value is below zero, its corresponding lens has a poorly clustered structure. The classification does not necessarily have physical meaning as a data subdivision. ${ }^{34}$

Another point to be addressed is the multilabel classification problem as a sample can belong to more than one class. ${ }^{35}$ In this study, the performance measure is selected as the $F$-measure, which combines precision and recall into a scalar, ${ }^{36}$ and is estimated from a multilabel data set as follows:

$$
\operatorname{pre}\left(q, t_{r}\right)=\frac{T P_{k}}{T P_{k}+F P_{k}}=\frac{N_{q t_{r}}}{N_{q}} \text { and } \operatorname{rec}\left(q, t_{r}\right)=\frac{T P_{k}}{T P_{k}+F N_{k}}=\frac{N_{q t_{r}}}{N_{t_{r}}},
$$

respectively, where $T P_{k}$ (true positive) is the number of samples that are correctly labeled as belonging to the $k^{\prime}$ th class, and false positive $\left(F P_{k}\right)$ and false negative $\left(F N_{k}\right)$ are defined analogously; $N_{q t_{r}}$ is the number of lenses from original cavity $q$ in cluster $t_{r} ; N_{t_{r}}$ denotes the total number of lenses in cluster $t_{r}$; and $N_{q}$ represents the number of lenses from the mold cavity $q$. The $F$-measure is weighted by a parameter $\beta^{2} \in(0,+\infty]$. Then, the overall performance in the $N$ categories can be computed using the micro-averaged $F$-measure, which is defined as follows: $:^{37,38}$

$$
F_{\beta}^{q}=\frac{1+\beta^{2}}{\left(1+\beta^{2}\right)+\frac{\sum_{k=1}^{N}\left(F P_{k}+\beta^{2} F N_{k}\right)}{\sum_{k=1}^{N} T P_{k}}} .
$$

To obtain the clustering results, the $F 1$-measure parameters for a single cavity $\beta=1$, then the total $F$-measure $F$ for the typical Zernike terms is obtained from the weighted average values of each category, $q$, as follows: 


$$
F=\sum_{c} \frac{\mathrm{N}_{q} \times F_{\beta}^{q}}{\sum_{q} \mathrm{~N}_{q}} .
$$

$F$ lies between 0 and 1 . In this way, it is expected that the typical Zernike terms have a typical spatial distribution. Thus, the number of lenses from the original cavity $q$ in cluster $t_{r}$, which possesses the typical Zernike term, must be the same as the number of lenses in the corresponding mold cavity $q$. Further, it must be the same as those of the clusters in class $t_{r}$, achieving $N_{q t_{r}}=N_{q}=N_{t_{r}}, F=F(q)=1$. When $F$ is closer to 1 , a typical term in the wavefront aberration representation is selected, indicating that it is more sensitive to the errors of different mold cavities. In contrast, a value of 0 indicates poor classification.

\section{Extraction of Typical Wavefront Aberration Terms in the S-Line Measurement}

As discussed above, F-FCMs are introduced to extract typical aberration terms for small training data sets (small molds and corresponding lenses). Seven different mold cavities were chosen for the $\mathrm{S}$-line experiments, labeled as 2BA, 3BA, 4BA, 6BA, 7BA, 8BA, and 9BA according to their TWP in the M-line. Table 1 gives the label and the TWP of mold cavities and lenses used in the experiments. For each mold cavity, 20 lenses were selected by uniform random sampling and characterized for datasets; in total, 140 lenses were collected as samples to assess typical aberrations in the S-line.

Each lens was measured in the SHWFS system to obtain the 36 Zernike polynomial coefficients of the wavefront, namely, $Z_{k}$. The measurement matrix $\mathrm{MES}_{140 \times 36}$ was obtained from these 140 lenses. Each row of the matrix represented 36 aberration coefficients of one lens. We randomly selected $r$ lenses as the initial centroids for one run of the algorithm in Eqs. (5) and (6). Two stopping criteria were applied in the algorithm before convergence. As stated previously, $\varepsilon$ denotes the error between two consecutive values of the constrained objective function, and $l_{M x}$ represents the prespecified maximum number of iterations.

About 10 runs of the FCM algorithm were performed. One run of the FCM algorithm led to a local solution. Employing the local optimization method, the FCM algorithm was run with different initializations to enable exploration of the entire data space and obtain an optimal solution. The membership values and centroids that correspond to the lowest criterion value were retained. The parameters used in the calculations are given in Table 2.

Table 1 Number of mold cavities and lenses used in experiments.

\begin{tabular}{lccc}
\hline \hline No & Mold & Input $\left(N_{i}\right)$ & Total working performance \\
\hline 1 & 2BA & 20 & $93 \%$ \\
2 & 3BA & 20 & $92 \%$ \\
3 & 4BA & 20 & Trial group \\
4 & 6BA & 20 & $87 \%$ \\
5 & 7BA & 20 & $67 \%$ \\
6 & 8BA & 20 & NG part \\
7 & $9 B A$ & 20 & Trial group \\
\hline \hline
\end{tabular}

Table 2 Parameters of FCM algorithm.

\begin{tabular}{cccccc}
\hline \hline$Z_{i}(\lambda)$ & $N$ & $r$ & $m$ & $\varepsilon$ & $I_{M x}$ \\
\hline & 140 & 7 & 2 & $10^{-7}$ & 500 \\
\hline \hline
\end{tabular}




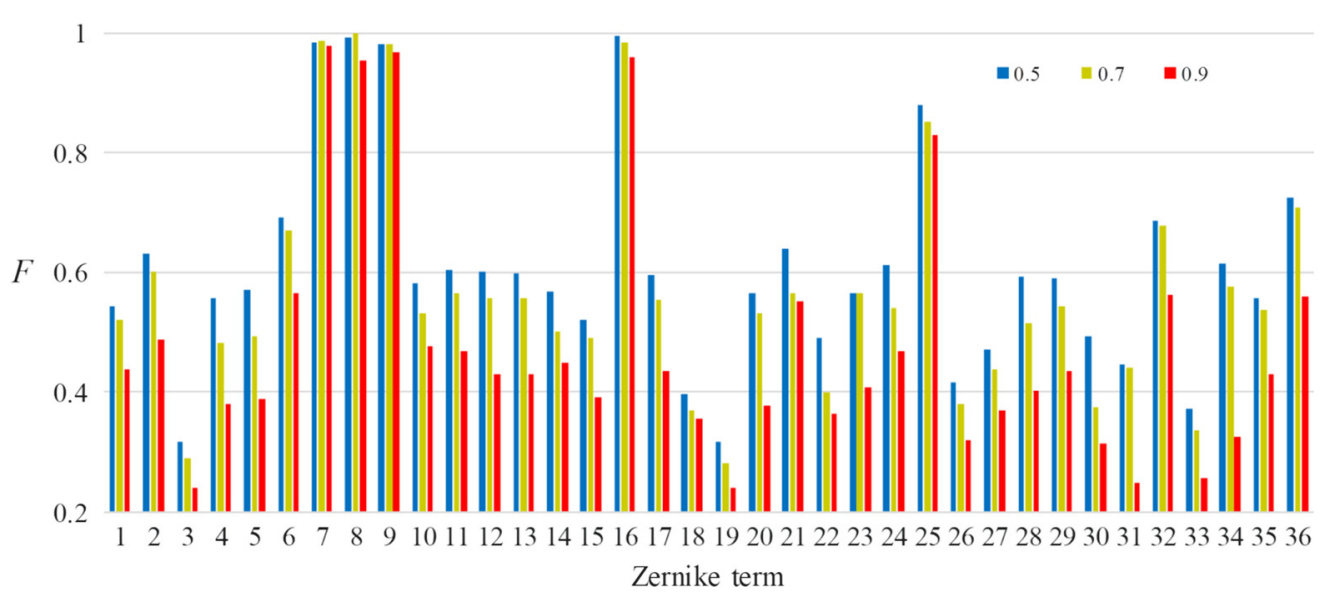

Fig. 4 F-distribution analysis for various Zernike terms with different membership functions.

\subsection{Selection of Zernike Terms Closely Associated with Clusters}

For all Zernike terms, i.e., $\mathrm{MES}_{140 \times 36}$, fuzzy cluster analysis was conducted for each column. Accordingly, the $F$-measure parameters were obtained experimentally from all 140 samples. The 36 terms, $Z_{k}(k=1,2, \ldots 36)$, were chosen as the $x$ axis. The $y$ axis was based on different $F$-measures with a variation range of $\mu_{\text {Threshold }}$ (threshold of $u_{k i}$ using $0.5,0.7$, and 0.9 ), resulting in an $F$ distribution of $(0.5,0.7$, and 0.9$)$, as shown in Fig. 4.

According to the results shown in Fig. 4 , the typical Zernike terms, i.e., $Z_{7}, Z_{8}, Z_{9}$, and $Z_{16}$, can achieve the ideal case of $F \approx 0.8$, even when adopting different values of $\mu_{\text {Threshold }}$. Consequently, the typical terms are expected to classify lenses with different fabrication errors from different mold cavities. Meanwhile, a smaller $F$ value suggests a sensitive term, e.g., $Z_{25}$, and a scattered error distribution when it is applied.

\subsection{Distributions of Membership and Silhouette Values}

As deduced from Fig. 4, the wavefront data set can be divided into four categories: terms that are not sensitive, such as $Z_{4}, Z_{5}$, and $Z_{10}$; low-order terms (third-order) that are sensitive, such as $Z_{7}, Z_{8}$, and $Z_{9}$; high-order terms (fifth-order) that are sensitive, such as $Z_{16}$; and terms that are relatively sensitive, such as $Z_{25}$. The scatterplots in Fig. 5 show the highest (horizontal axis) and second-highest (vertical axis) $\mu_{k i}$ values for $Z_{4}, Z_{7}, Z_{16}$, and $Z_{25}$. The above observations suggest that it may be relevant to restrict the clustering algorithm to the typical Zernike terms that show strong associations to a certain cluster.

For further investigation of a lens selection method that leads to clusters with better separation, the silhouette values were calculated for each lens within each cluster. All lenses were accounted for, i.e., the lenses with higher maximum $\mu_{k i}$ values were included as well. Boxplots of the silhouette values are shown for each cluster in Fig. 6. As expected, the sensitive terms, in which the coefficient increases and approaches one, have significantly higher silhouette values for the given cluster. In the case of $Z_{4}$, the entire cluster, which contains numerous lenses with negative silhouette values without lens selection, is a poorly clustered structure. Meanwhile, $Z_{7}$, a cluster that has no negative silhouette value for any selected lens, is one of the clusters with the best separation [Fig. 6(b)]. In contrast, for the cases of $Z_{16}$ and $Z_{25}$, negative values start to appear in one cluster (such as cluster 6 of $Z_{16}$ and cluster 3 of $Z_{25}$ ), indicating that the distribution may be scattered under the current injection-molded lens error distribution.

As shown in Fig. 5, the distribution of the membership values varies markedly from one term to another. For $Z_{7}$ (similarly to $Z_{8}$ and $Z_{9}$ ), the behavior of each lens can be approximately determined by its first and second membership values (maximum $\mu_{k i}>0.9$ ) or the third-order term, indicating that the current fabrication error mainly influences the third-order term. In contrast, in the case of $Z_{4}$ (similarly to $Z_{5}$ and other aberrations), the distribution of the $\mu_{k i}$ values is higher, and some lenses $(\approx 8 \%)$ exhibit loose associations with any cluster (maximum $\mu_{k i}<0.5$ ). For $Z_{16}$, the fraction of lenses with maximum $\mu_{k i}$ values larger than 0.9 is markedly higher than 
Cheng et al.: Fabrication-error analysis of injection-molded aspheric elements...

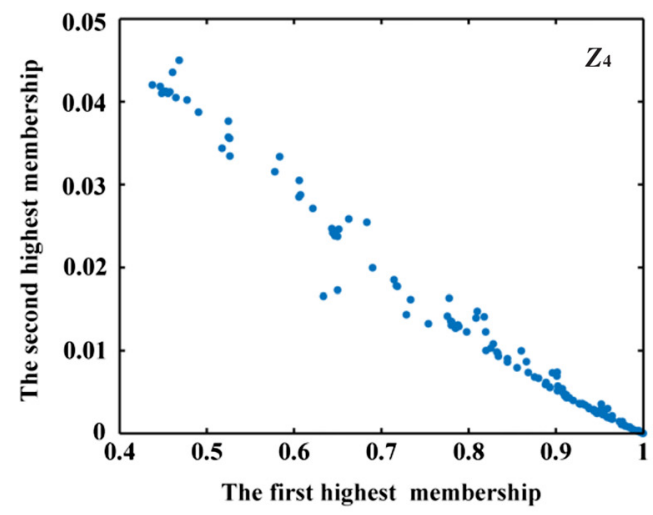

(a)

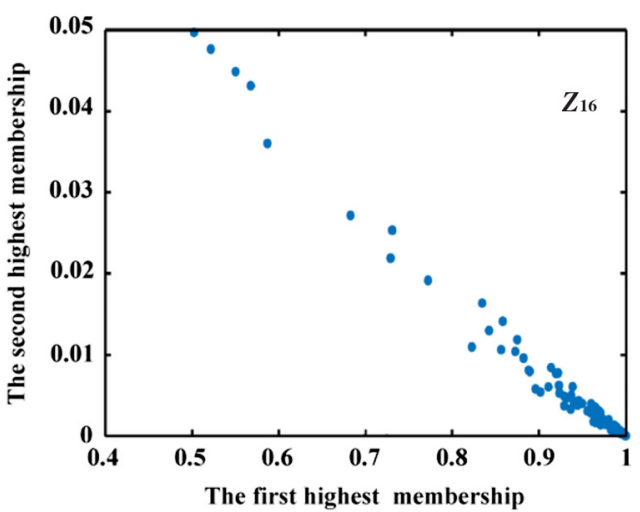

(c)

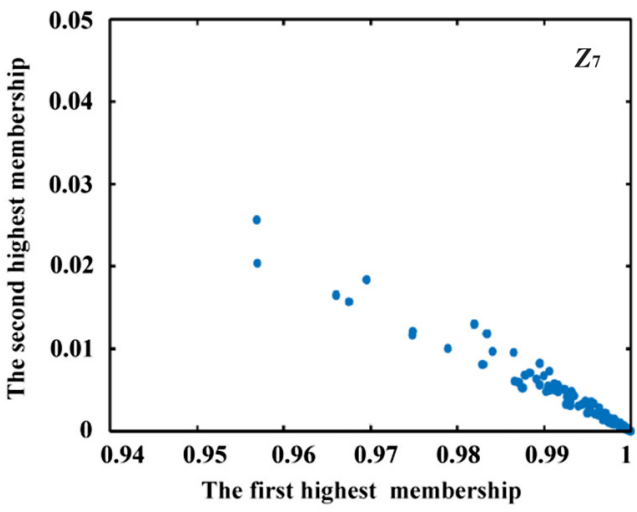

(b)

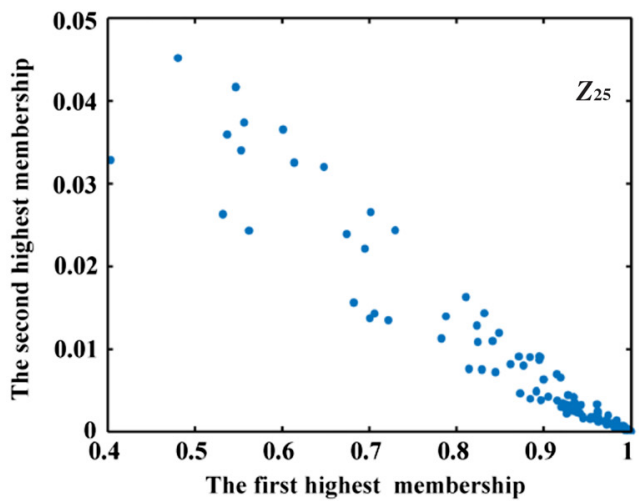

(d)

Fig. 5 Scatterplot of two highest membership values of all lenses in four categories of sets. The vertical lines correspond to the median highest membership values: (a) $Z_{4}$; (b) $Z_{7}$; (c) $Z_{16}$; and (d) $Z_{25}$ terms.

for the other terms $(\approx 85 \%)$, and a fraction with maximum $\mu_{k i}$ values below 0.5 does not exist. For $Z_{25}$, the fraction of the lenses with maximum $\mu_{k i}$ values larger than 0.9 is $\approx 75 \%$, and the proportion with maximum $\mu_{k i}$ values $<0.5$ is $\approx 1.5 \%$.

In Fig. 6, it shows that the sensitivity to fabrication errors decreases as the term order increases and the sensitive aberration terms have better cluster structures. Moreover, it implies that the Zernike terms in the transmitted wavefront that are related to the SA, coma aberration, and astigmatism are predominantly sensitive. The Zernike terms related to the third-order astigmatism are $Z_{5}$ and $Z_{6}$, which are not predominantly sensitive. It is assumed that different errors have a cross-effect on the correction of astigmatism aberrations, and that the coherent relationship of the aberrations to the errors is not monotonic or more complicated.

\section{Results and Discussions}

\subsection{Typical Wavefront Aberration Terms for Performance Evaluation of Mold Cavities}

Figure 7 shows the $Z_{7}$ and $Z_{8}$ distributions for sampling dataset from distinct mold cavities. Figure 8 shows the $Z_{9}, Z_{16}$, and $Z_{25}$ distributions, which indicates that $Z_{9}$ and $Z_{16}$ distributions are more concentrated, and the distribution begins to disperse. Nevertheless, for different lenses from a certain mold cavity, $Z_{25}$ can still be distinguished. $Z_{7}, Z_{8}, Z_{9}, Z_{16}$, and $Z_{25}$ are the terms related to the third-order coma aberrations and third-, fifth-, and seventh-order SAs, respectively.

The error estimates of the lenses from different mold cavities are predominantly manifested as the third-order coma aberrations and third-, fifth-, and seventh-order SAs, which nevertheless show significant effects. In addition to the multi-order terms of the SA introduced by the 


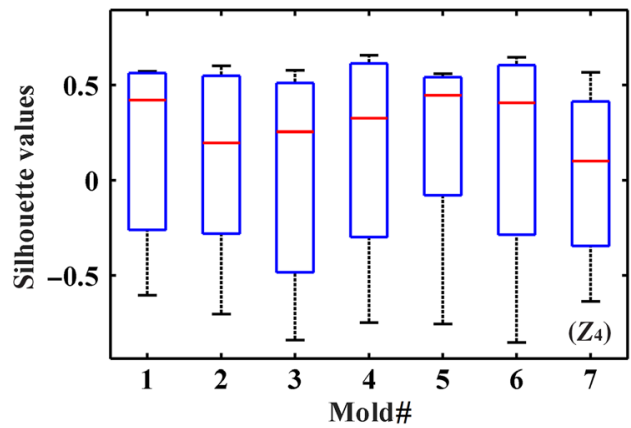

(a)

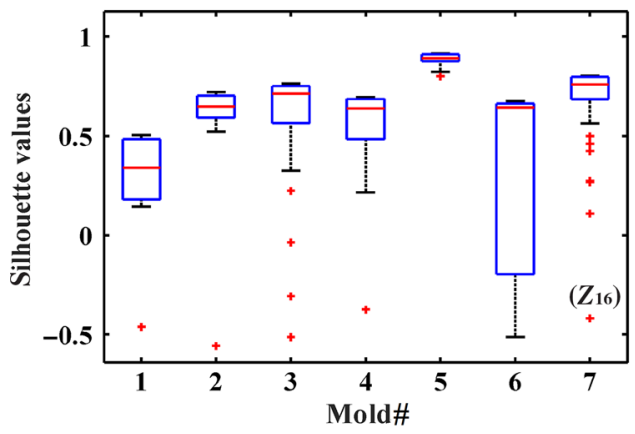

(c)

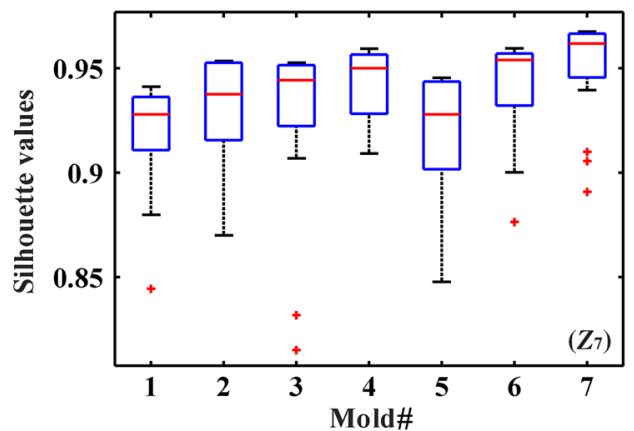

(b)

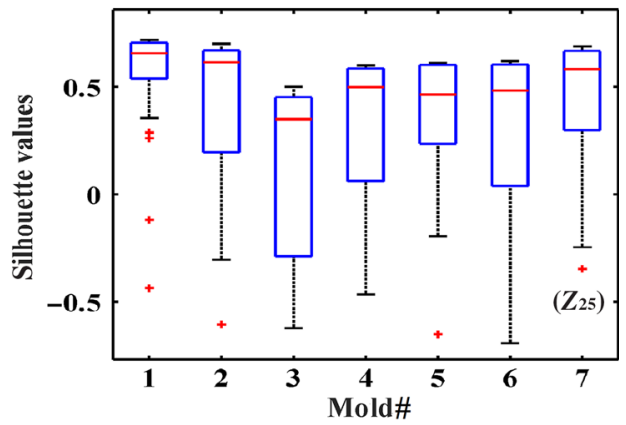

(d)

Fig. 6 Boxplots of silhouette values of lenses in clusters. For each lens, a silhouette value was computed with a threshold of 0.5 for the (a) $Z_{4}$; (b) $Z_{7}$; (c) $Z_{16}$; and (d) $Z_{25}$ terms.

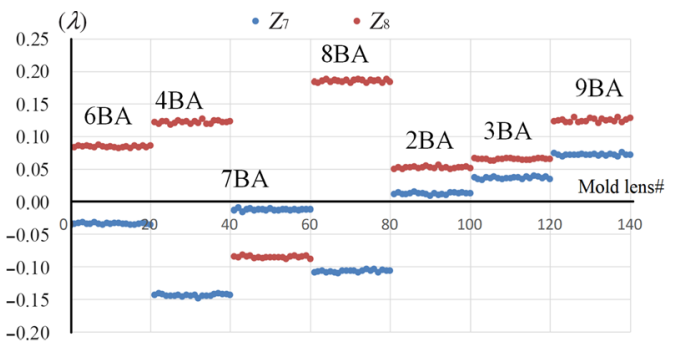

(a)

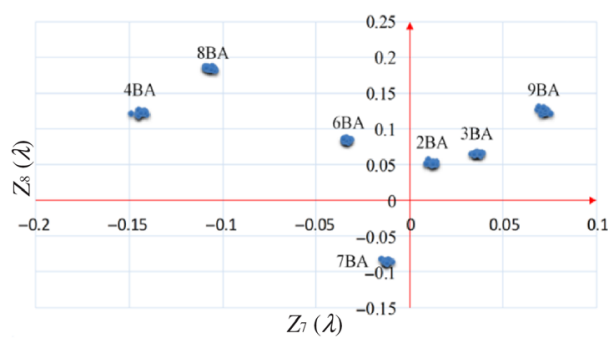

(b)

Fig. 7 Distributions of $Z_{7}$ and $Z_{8}$ terms of wavefront polynomial for sampling dataset. (a) Individual distributions with individual variations of $Z_{7}$ and $Z_{8}$. (b) Joint distribution of these two coefficients, where the $x$ axis is $Z_{7}$ and the $y$ axis is $Z_{8}$.

injection-molding fabrication error, the system image quality is largely compromised by field-ofview-related aberrations, specifically, coma aberrations. Figures 7(b), 8(a), and 8(b) indicate that, by considering $Z_{7}$ and $Z_{8}$ or $Z_{9}$ and $Z_{16}$ together, the lenses from different mold cavities can be separated; Fig. 8(d) shows that mold cavities labeled as $2 \mathrm{BA}$ and $3 \mathrm{BA}$ with the highest value of TWP, or $93 \%$ and $92 \%$, respectively, have been separated distinctly from other mold cavities.

\subsection{Performance Characterization with the I-Line and P-Line Measuring Results}

Further, these extracted typical terms were characterized in the evaluation experiments. Lenses from another five different mold cavities were selected for a general applicability discussion, and they are labeled as $4 \mathrm{AB}, 4 \mathrm{AR}, 5 \mathrm{BA}, 8 \mathrm{AR}$, and $11 \mathrm{AR}$. The extracted typical aberration terms in the $\mathrm{S}$-line would be used to allow a valid characterization among the comparison results of the I-line and P-line. 


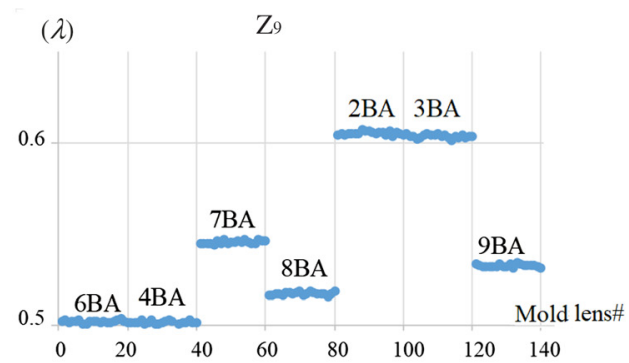

(a)

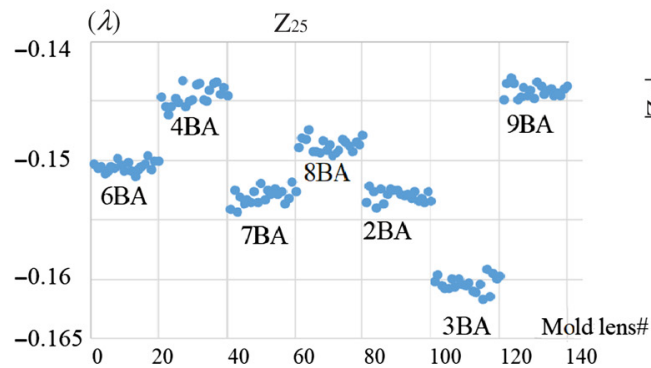

(c)

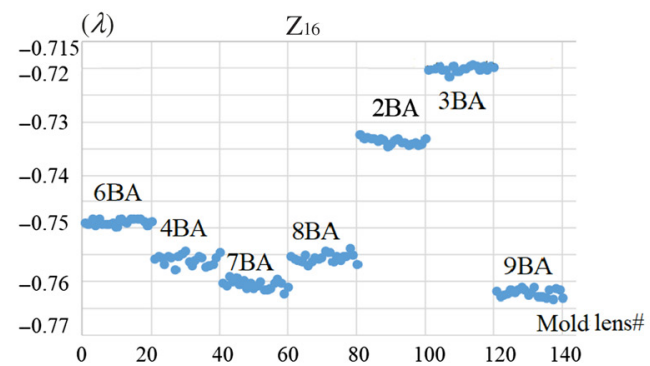

(b)

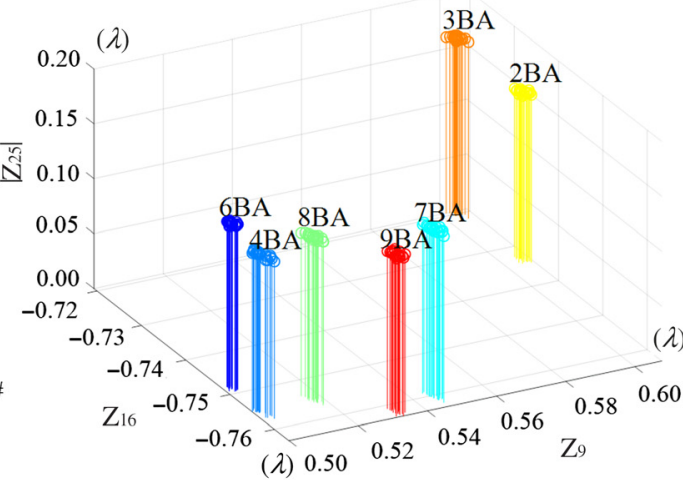

(d)

Fig. 8 Distribution analyses with individual variations of (a) $Z_{9}$; (b) $Z_{16}$; and (c) $Z_{25}$. (d) Joint distribution analysis of $Z_{9}(\lambda), Z_{16}(\lambda)$, and $\left|Z_{25}\right|(\lambda)$.

Tables 3 and 4 give the I-line and P-line measurement results, the surface profiles of two sides of the element, respectively. Their TWP are also given, as defined in Sec. 2.2. First, as list in Tables 3 and 4, lens fabrication errors for the two side surfaces [A1 and A2 in Fig. 1(a)] were indicated with the PV and RMS values. Although the differences in the PV and RMS values for the surface errors corresponding to the other four mold cavities are small, the differences are large compared to those for 5BA. This finding suggests that, when fabricating the lenses, the fabrication errors caused during the injection-molding procedure were significantly different between 5BA (values typed in italic font) and the four other mold cavities. Meanwhile, the error values of the other four lenses are closer to each other and larger.

Further, in this study, in consideration of the relationship between the wavefront SA and the foci location, a model-fitting analysis of the aspheric coefficient $R_{0}$ of surface profile of the optical element was performed. For aspheric surfaces (ISO 10110, International Organization for Standardization, Switzerland):

Table 3 I-line measurement results ( $\mu \mathrm{m}$; L1_A1 and L1_A2 represent the two sides).

\begin{tabular}{|c|c|c|c|c|c|c|c|c|c|}
\hline \multirow[b]{3}{*}{ Mold } & \multicolumn{4}{|c|}{ L1_A1 } & \multicolumn{4}{|c|}{ L1_A2 } & \multirow{3}{*}{$\begin{array}{c}\text { Total working } \\
\text { performance }(\%)\end{array}$} \\
\hline & \multicolumn{2}{|c|}{ Vertical $(x)$} & \multicolumn{2}{|c|}{ Horizontal $(y)$} & \multicolumn{2}{|c|}{ Vertical $(x)$} & \multicolumn{2}{|c|}{ Horizontal $(y)$} & \\
\hline & PV & RMS & PV & RMS & PV & RMS & PV & RMS & \\
\hline $4 \mathrm{AB}$ & 0.278 & 0.050 & 0.257 & 0.049 & 0.465 & 0.119 & 0.551 & 0.129 & 78.9 \\
\hline 4AR & 0.299 & 0.074 & 0.361 & 0.081 & 0.654 & 0.153 & 0.807 & 0.168 & 76.4 \\
\hline $5 B A$ & 0.036 & 0.009 & 0.137 & 0.019 & 0.186 & 0.066 & 0.152 & 0.063 & 95.1 \\
\hline $8 A R$ & 0.246 & 0.048 & 0.274 & 0.052 & 0.517 & 0.139 & 0.626 & 0.148 & 80 \\
\hline $11 \mathrm{AR}$ & 0.213 & 0.049 & 0.288 & 0.059 & 0.658 & 0.187 & 0.818 & 0.203 & 83.5 \\
\hline
\end{tabular}


Cheng et al.: Fabrication-error analysis of injection-molded aspheric elements...

Table 4 P-line measurement results ( $\mu \mathrm{m}$; L1_A1 and L1_A2 represent the two sides).

\begin{tabular}{|c|c|c|c|c|c|c|c|c|c|}
\hline \multirow[b]{3}{*}{ Mold } & \multicolumn{4}{|c|}{ L1_A1 } & \multicolumn{4}{|c|}{ L1_A2 } & \multirow{3}{*}{$\begin{array}{c}\text { Total working } \\
\text { performance (\%) }\end{array}$} \\
\hline & \multicolumn{2}{|c|}{ Vertical $(x)$} & \multicolumn{2}{|c|}{ Horizontal $(y)$} & \multicolumn{2}{|c|}{ Vertical $(x)$} & \multicolumn{2}{|c|}{ Horizontal $(y)$} & \\
\hline & PV & RMS & PV & RMS & PV & RMS & PV & RMS & \\
\hline $4 A B$ & 0.2593 & 0.0487 & 0.2481 & 0.0457 & 0.4463 & 0.0997 & 0.4996 & 0.1178 & 78.9 \\
\hline $4 \mathrm{AR}$ & 0.3099 & 0.0601 & 0.3338 & 0.0672 & 0.5545 & 0.1113 & 0.6114 & 0.1392 & 76.4 \\
\hline $5 B A$ & 0.0816 & 0.0233 & 0.0948 & 0.0289 & 0.2028 & 0.0520 & 0.1321 & 0.0358 & 95.1 \\
\hline $8 A R$ & 0.2019 & 0.0532 & 0.2390 & 0.0468 & 0.4946 & 0.1062 & 0.5627 & 0.1356 & 80 \\
\hline $11 \mathrm{AR}$ & 0.2015 & 0.0491 & 0.2592 & 0.0524 & 0.6041 & 0.1360 & 0.7046 & 0.176 & 83.5 \\
\hline
\end{tabular}

$$
x=\frac{c y^{2}}{1+\sqrt{1-(K+1) c^{2} y^{2}}}+\sum_{j=0} A_{j} y^{2 j+4},
$$

where $c=1 / R_{0}$ is the vertex curvature, $K$ denotes the conic constant, and $A_{j}$ is the coefficient. If this expression includes only the first term on the right, it indicates a strict conic surface. In Eq. (11), when fitting rotational symmetric surfaces with nonorthogonal polynomials using the least squares method, the values of the polynomial coefficients increase as the polynomial order increases, such that massive coefficients may be derived in some cases. These coefficients are usually randomly oriented ${ }^{26}$ with a tendency of alternating positive and negative values in the higher orders. Thus, a suitable parameter should be used to describe the features of the surface deviation, and this feature is employed for correlation with the SA terms. Furthermore, the lowerorder parameter has the largest influence on all higher-order terms; thus, the conic parameter was chosen in this study. Let $x$ be expressed in terms of $y^{2}$. Then, the conic part in the polynomial becomes an infinite series arranged in ascending powers of $y^{2}$, and the polynomial can be expressed as follows:

$$
x=\frac{1}{2 R_{0}} y^{2}+\left(\frac{\left(1-e^{2}\right)+A_{0}}{8 R_{0}^{3}}\right) y^{4}+\left(\frac{\left(1-e^{2}\right)^{2}+A_{1}}{16 R_{0}^{5}}\right) y^{6}+\cdots=\sum_{j=1} B_{j} y^{2 j} .
$$

Here, $R_{0}$ is the basic parameter and the higher orders are linear correction parameters on a lower-order basis. Then, $R_{0}$ is stable as the polynomial coefficient and could be used to analyze the surface deviation characteristics and its imaging performance on the SA terms. In Eq. (12), coefficient $B_{j}, j=1 \cdots 6$ was fitted to the data obtained in the P-line measurement. We defined $K_{j}=\widehat{B}_{j}-B_{j}$, which indicates the deviation between the design value, $B_{j}$, and measured value, $\widehat{B}_{j}$. Thus, the values of $K_{1}$ for different mold cavities are calculated and the results in Fig. 9 reveal that the mold cavity $5 \mathrm{BA}$ is especially better and different from the others, which reach the same conclusion as those in Tables 3 and 4 .

The transmitted wavefront measurement results in the proposed S-line are shown in Fig. 10. It shows that the lens wavefronts from the different mold cavities exhibit very similar patterns with minimal differences in the RMS values for the overall wavefront. Moreover, the RMS differences are below $0.01 \lambda$. Thus, in terms of the overall wavefront analysis, it is difficult to characterize the optical performance using the PV parameter of the transmitted wavefront. Nevertheless, it is convenient and effective to distinguish the optical performance by analyzing the typical terms (the different order of SA $Z_{9}, Z_{16}$, and $Z_{25}$ ), as shown in Fig. 10(f). The distribution of typical terms relative to the wavefront $S A$ reveals that the $5 \mathrm{BA}$ is quite different from those of the other cavities, i.e., 4AB, 4AR, 8AR, and 11AR, which appear more aggregated. These results are consistent with those of the corresponding mold cavities measured in the 


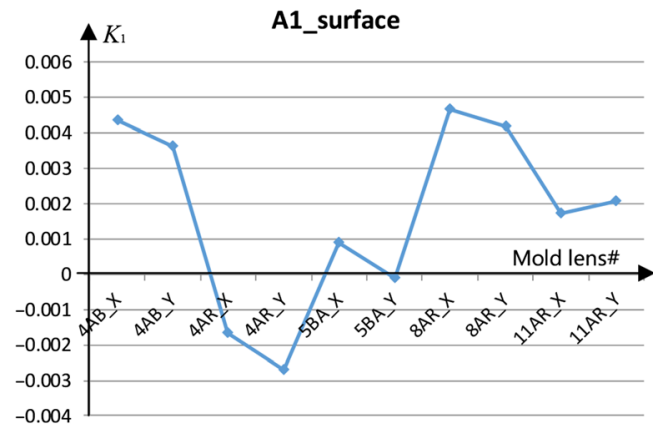

(a)

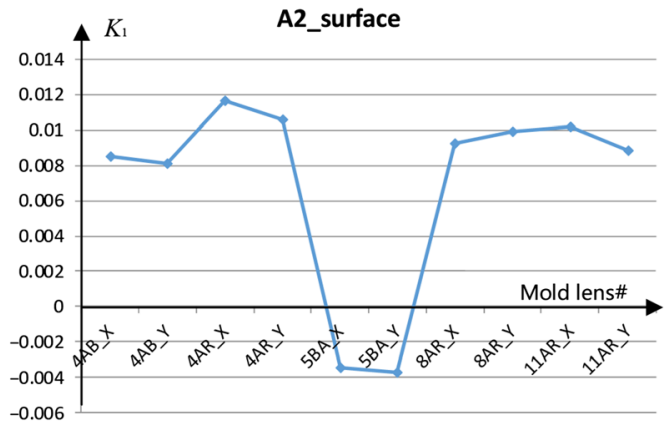

(b)

Fig. 9 Experimental results for $K_{1}$ from five mold cavities in P-line measurement [(a): $A 1$ surface; (b): A2 surface].

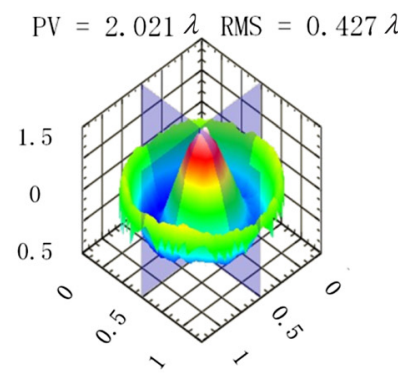

(a)

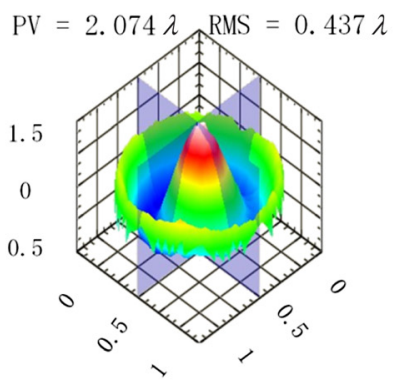

(b)

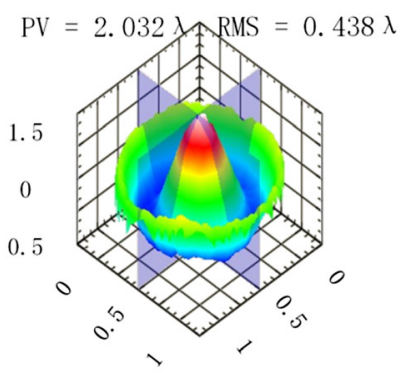

(c)

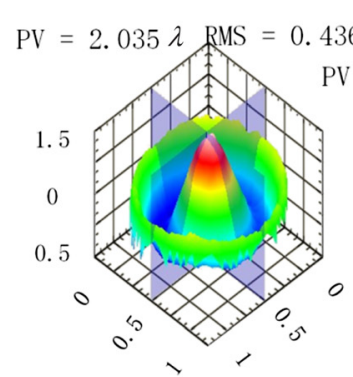

(d)

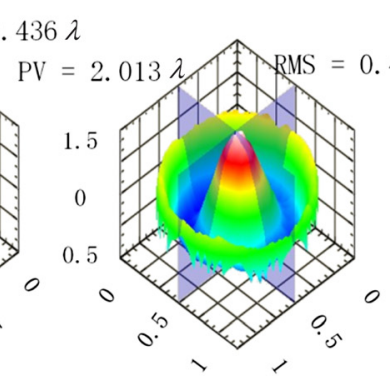

(e)

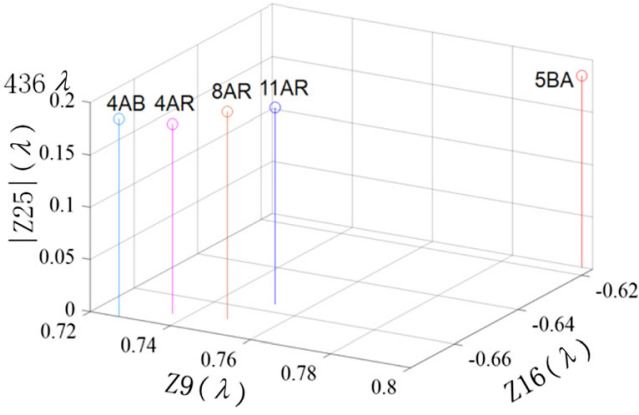

(f)

Fig. 10 SHWFS-measured lens wavefronts from different mold cavities: (a) 4AB; (b) 4AR; (c) 5BA; (d) 8AR; and (e) 11AR. (f) Distribution analysis for SA terms $Z_{9}, Z_{16}$, and $Z_{25}$ from different mold cavities.

P-line and I-line. Therefore, the results show that, for S-line measurement, details such as the error distribution form of the typical aberration terms could be properly used for error evaluation.

\subsection{Relationship Between Decenter of Two Aspherical Surface and Coma Aberration Term}

In addition to surface errors, S-line measurement results are also used to analyze the decenter error between the two surface sides of the element, which could exist in the fabrication process of injection-molding. First, we check the decenter errors and their wavefront errors in the simulation software (CODE V 11.2, Synopsys, Inc., Pasadena, California). In the numerical model, the experimental system (Fig. 2) was simplified by removing the focusing and beam-expanding assemblies. The effective aperture was set to $1 \mathrm{~mm}$, and the results are shown in Fig. 11. The influence of the lens thickness was not considered. The surface center deviation errors along 


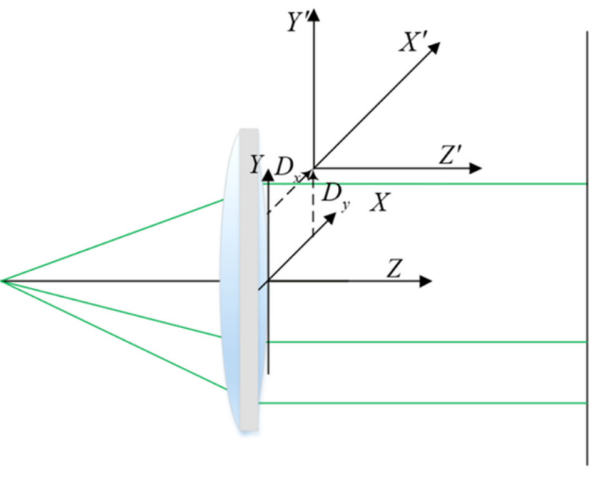

(a)

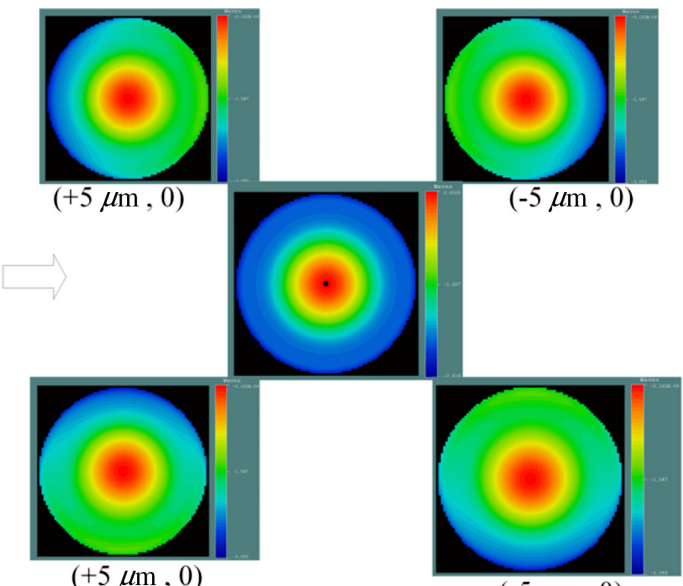

$(-5 \mu \mathrm{m}, 0)$

(b)

Fig. 11 (a) Schematic diagram of simulated light path. The basic coordinate system of each surface is a right-handed coordinate system, where the $z$ axis is defined as the optical axis direction, and the aspherical surface is oriented in relation to the $z$ axis. (b) Simulation analysis of the decenter. The center in (b) is the standard wavefront, the upper left in (b) is the $x$ axis $5 \mu \mathrm{m}$ eccentric wavefront, and the upper right in (b) is the $x$ axis $-5 \mu \mathrm{m}$ eccentric wavefront. The lower left is the $y$ axis $5 \mu \mathrm{m}$ eccentric wavefront, and the lower right is the $y$ axis $-5 \mu \mathrm{m}$ eccentric wavefront. (the boxes in the figure are squares of $1-\mathrm{mm}$ side length.).

the $x$ and $y$ axes, caused by the mold overturning or lateral movement during the mold-clamping process, were identified and simulated. In the injection-molding process, the actual decenter error ranged from -30 to $30 \mu \mathrm{m}$. The resulting wavefront changes are shown in Fig. 11(b). As shown in Fig. 12, the simulation results show that $Z_{7}$ and $Z_{8}$ are linearly changed as the decenter increases, indicating a linear relationship between the coma aberration with decenter and the corresponding lens.

These simulation results were found to be consistent with the experimental results of the decenter detection test. The aspheric elements of samples from these seven mold cavities (2BA, 3BA, 4BA, 6BA, 7BA, 8BA, and 9BA), were measured in the S-line and P-line. Their Zernike terms relative to the coma and decenter along the $x$ and $y$ axes were evaluated. A statistical model for the third-order coma aberrations $\left(Z_{7}, Z_{8}\right)$ measured in S-line and the decenter error measured in P-line was constructed by linear regression. The regression validity was evaluated using the correlation coefficient $R^{2}$, as expressed in Eq. (13). Then, linear regression analysis was performed, and the results in Eq. (14) were obtained, indicating a good linear fit (Fig. 13).

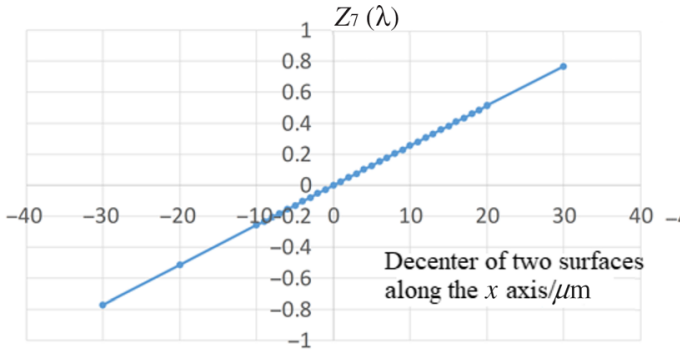

(a)

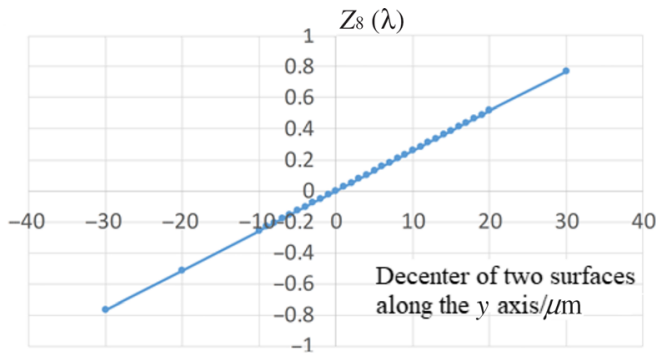

(b)

Fig. 12 Simulation results for the relationship between the decenter and third-order coma in the optical element. (a) Simulated $x$ axis decenter and third-order coma aberration $Z_{7}$. (b) Simulated $y$ axis decenter and third-order coma aberration $Z_{8}$. 


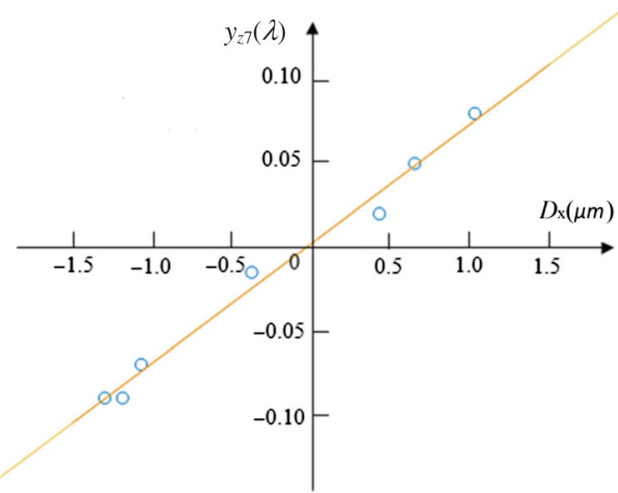

(a)

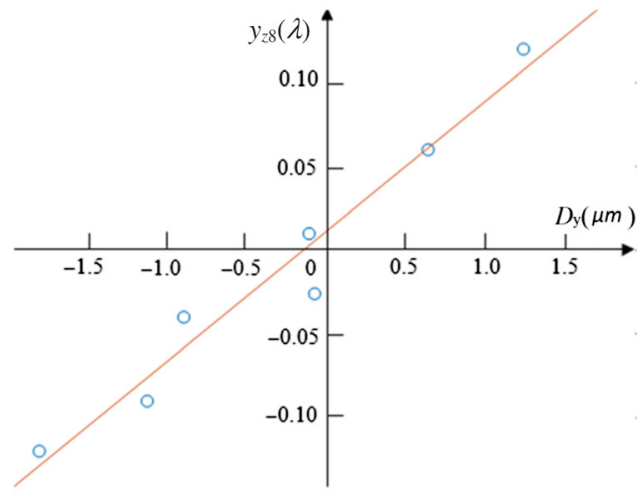

(b)

Fig. 13 Eccentricity effects and corresponding fitting analysis. (a) Fitting analysis results for $x$ axis decenter and third-order coma aberration $Z_{7}$. (b) Fitting analysis results for $y$ axis decenter and third-order coma aberration $Z_{8}$.

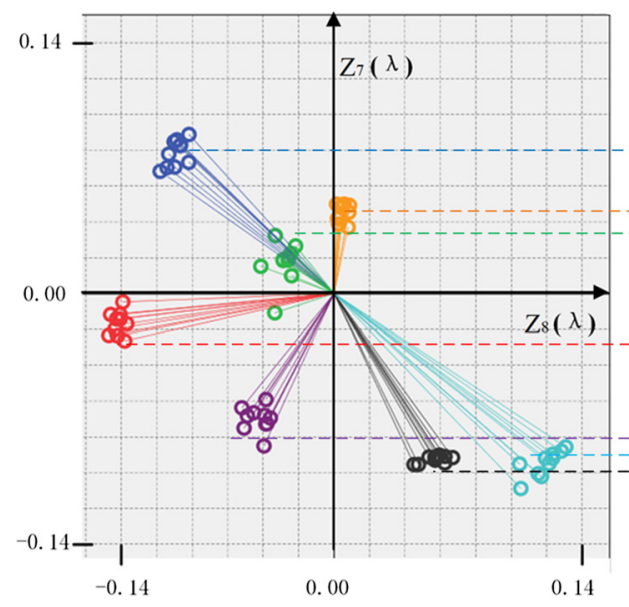

(a)

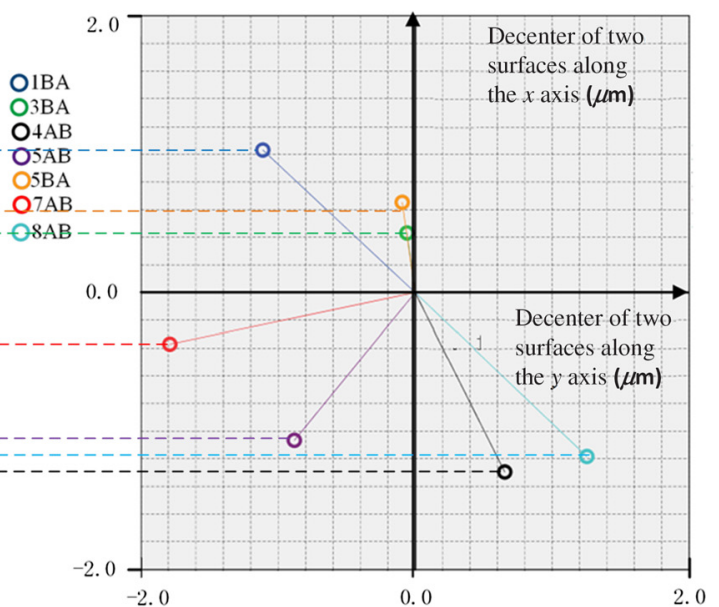

(b)

Fig. 14 Two-dimensional mapping (indicated with the dotted lines) for measured coma aberration terms $Z_{7}$ and $Z_{8}$ (a) using SHWFS and decenter of two aspherical surfaces (b) using the profilometer.

The relationship was further confirmed in a two-dimensional mapping of the results measured in the S-line and P-line, as shown in Fig. 14, which reveals that an approximately linear correlation exists between the third-order coma and the decenter of the lens.

$$
\begin{aligned}
& R^{2}=\frac{\text { Sum squared residual }(\mathrm{SSR})}{\text { Sum squared total }(\mathrm{SST})}=1-\frac{\text { Sum squared error }(\mathrm{SSE})}{\text { Sum squared total }(\mathrm{SST})}, \\
& y_{Z 8}=0.0776596858483021 D_{y}+0.0102023118878652 R^{2}=0.9878, \\
& y_{Z 7}=0.0713977936741716 D_{x}-0.00197165997012183 R^{2}=0.9567 .
\end{aligned}
$$

\section{Conclusions}

Aspheric-lens fabrication errors were analyzed in transmission optical paths with a wavefrontsensing technique to estimate the optical component errors, focusing on the errors caused during injection molding. Accordingly, transmitted wavefront analysis and extraction of typical 
aberration terms were performed to evaluate injection-molded aspheric lenses. Mobile-phone camera lenses, primarily exhibiting spherical and coma aberrations, were used as samples. The typical Zernike terms in the transmitted wavefront aberration function were evaluated using unsupervised-learning-based F-FCMs. This indicates that the fabrication errors caused during the injection-molding procedure differ significantly, and the extracted typical aberration terms thus allow a valid characterization by comparison of the results obtained with a profilometer, interferometer, and the SHWFS. Mapping relationships between the measurement results reveal the correlation between the third-order coma and the decenter of the lens.

The experimental results show that interferometers and profilometers could ensure high resolutions in the sub-nanometer range, however, it would take more than $5 \mathrm{~min}$ to complete the measurements for each lens. Further, it is necessary to measure the two faces of each aspheric lens separately when measuring the reflective wavefront of a surface. Currently, SHWFS method could not achieve a measurement accuracy as high as that obtainable by interferometry. However, using the transmitted wavefront measurement with SHWFS technique, combined with the proposed aberration term-based fabrication-error analysis method in this study, has shown that a lens can be measured in a single test trial to obtain the complete error state and the analysis of the transmitted wavefront could serve as a relatively low-cost general-purpose method in the production line.

The extracted typical Zernike terms that were identified in the SHWFS measurements had shown their efficiency and accuracy when being employed as indicators for assessing surface and decenter errors. These terms can be applied to efficiently adjust the injectionmolding process conditions. This capability is critical for developing guidelines for error compensation during fabrication, especially for mass production of aspheric lenses via injectionmolding with multiple related factors. Based on the error distributions and principles of the corresponding variations in actual production, the ranges of typical factors can be determined. Subsequently, the expression can be used to make adjustments to fix specific fabrication errors for optical performance improvement of certain optical properties. Moreover, preliminary lens screening can be realized before performing higher-resolution examinations via interferometry and profilometry, and accordingly, more timely feedback for processing improvements can be provided.

In future studies, typical aberration terms in the SHWFS measurements will be discussed further, which is expected to provide additional information about the transmitted wavefront data, including surface defects, refractive indices, inhomogeneities, and other defects. Further, the inspection for the off-axis aberrations and the light spectra influence will be discussed so that a complete justification of the lens performance is realized.

\section{Appendices}

\subsection{Appendix A: Zernike Representation of the Wavefront Aberration Function}

Zernike polynomials provide an excellent metric basis for describing and understanding errors in optical surface geometry. In this study, coefficient indexing associated with the Zernike polynomial fringe form was employed. ${ }^{23}$ The measured wavefront was circular, and it was reconstructed by Zernike polynomial fitting. The first 36 terms of the fringe Zernike polynomial were selected to fit the wavefront in polar coordinates, $W(\rho, \theta)$, as follows:

$$
W(\rho, \theta)=\sum_{i=1}^{36} Z_{i} C_{i}(\rho, \theta)=Z_{1} C_{1}(\rho, \theta)+Z_{2} C_{2}(\rho, \theta)+\cdots,
$$

where $Z_{i}$ is the Zernike fitting coefficient; $C_{i}(\rho, \theta)$ is the Zernike orthogonal basis; and $\rho$ and $\theta$ are the two parameters of the polar coordinate system. These orthogonal bases satisfy orthogonality inside the unit circle. Figure 15 shows the Zernike polynomial. Table 5 shows the expressions for the fringe Zernike polynomial and descriptions of the corresponding aberrations. 


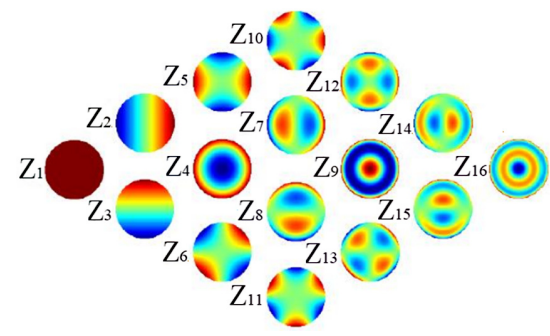

Fig. 15 Graphical depictions of Zernike polynomials.

Table 5 Physical meanings and expressions of Zernike polynomials.

\begin{tabular}{lccc}
\hline \hline Order & Term & Aberration description & Basis function \\
\hline $\mathrm{A}[0][0]$ & $Z_{1}$ & Piston or base & 1 \\
$\mathrm{~A}[1][-1]$ & $Z_{2}$ & Tilt $y$ & $R \cos (\theta)$ \\
$\mathrm{A}[1][1]$ & $Z_{3}$ & Tilt $x$ & $R \sin (\theta)$ \\
$\mathrm{A}[2][0]$ & $Z_{4}$ & Defocus & $2 R^{2}-1$ \\
$\mathrm{~A}[2][-2]$ & $Z_{5}$ & Astig $0^{\circ}$ or $90^{\circ}$ & $R^{2} \cos (2 \theta)$ \\
$\mathrm{A}[2][2]$ & $Z_{6}$ & Astig $\pm 45^{\circ}$ & $R^{2} \sin (2 \theta)$ \\
$\mathrm{A}[3][-1]$ & $Z_{7}$ & Coma $y$ & $\left(3 R^{3}-2 R\right) \cos (\theta)$ \\
$\mathrm{A}[3][1]$ & $Z_{8}$ & Coma $x$ & $\left(3 R^{3}-2 R\right) \sin (\theta)$ \\
$\mathrm{A}[4][0]$ & $Z_{9}$ & Primary spherical & $6 R^{4}-6 R^{2}+1$ \\
$\mathrm{~A}[3][-3]$ & $Z_{10}$ & Trefoil $y$ & $R^{3} \cos (3 \theta)$ \\
$\mathrm{A}[3][3]$ & $Z_{11}$ & Trefoil $x$ & $R^{3} \sin (3 \theta)$ \\
$\mathrm{A}[4][-2]$ & $Z_{12}$ & Secondary astigmatism $0^{\circ} / 90^{\circ}$ & $\left(4 R^{4}-3 R^{2}\right) \cos (2 \theta)$ \\
$\mathrm{A}[4][2]$ & $Z_{13}$ & Secondary astigmatism $\pm 45^{\circ}$ & $\left(4 R^{4}-3 R^{2}\right) \sin (2 \theta)$ \\
$\mathrm{A}[5][-1]$ & $Z_{14}$ & Secondary coma $y$ & $\left(10 R^{5}-12 R^{3}+3 R\right) \cos (\theta)$ \\
$\mathrm{A}[5][1]$ & $Z_{15}$ & Secondary coma $x$ & $\left(10 R^{5}-12 R^{3}+3 R\right) \sin (\theta)$ \\
$\mathrm{A}[6][0]$ & $Z_{16}$ & Secondary spherical & $20 R^{6}-30 R^{4}+12 R^{2}-1$ \\
\hline \hline
\end{tabular}

\subsection{Appendix B: Commercially Available Testing Equipment in Experiments}

The commercially available testing devices used in the error detection experiments were the Panasonic UA3P profilometer ${ }^{39}$ and Zygo Compass interferometer. ${ }^{40}$ The profilometer accuracy was $0.1 \mu \mathrm{m}$. The interferometer measurement accuracy was in the sub-nanometer range.

\subsection{Appendix C: Wavefront Measurement Data Reproducibility and Stability}

The experimental system was established based on the structure of the wavefront-sensing device from TriOptics, Germany. ${ }^{41}$ The detailed parameters of the components used in the testing system are shown in Table 6.

Table 6 Technical parameters of wavefront testing system.

\begin{tabular}{|c|c|c|c|c|c|c|c|c|}
\hline $\begin{array}{l}\text { Measurement } \\
\text { wavelength }\end{array}$ & $\begin{array}{l}\text { Test } \\
\text { lens }\end{array}$ & $\begin{array}{c}\text { Beam } \\
\text { expander }\end{array}$ & $\begin{array}{l}\text { Number } \\
\text { of micro } \\
\text { lenslets }\end{array}$ & $\begin{array}{l}\text { Aperture } \\
\text { dimension }\end{array}$ & $\begin{array}{c}\text { Tilt } \\
\text { dynamic } \\
\text { range }\end{array}$ & $\begin{array}{l}\text { Focus } \\
\text { dynamic } \\
\text { range }\end{array}$ & $\begin{array}{c}\text { Wavefront } \\
\text { measurement } \\
\text { absolute } \\
\text { accuracy (RMS) }\end{array}$ & $\begin{array}{l}\text { Repeatability } \\
\text { (RMS) }\end{array}$ \\
\hline $532 \mathrm{~nm}$ & $1 \mathrm{~mm}$ & $7.36 x$ & $150 \times 150$ & $15 \times 15 \mathrm{~mm}^{2}$ & $1500 \lambda$ & $500 \lambda$ & $\lambda / 20$ & $\lambda / 200$ \\
\hline
\end{tabular}




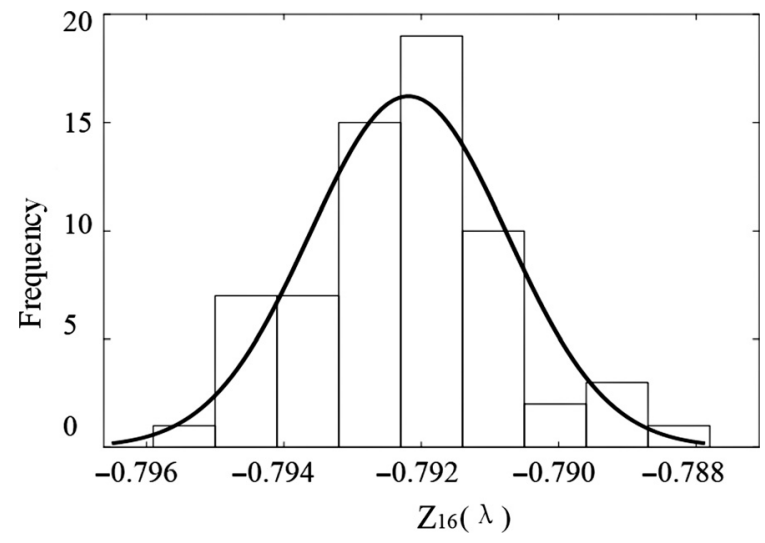

Fig. 16 Frequency distribution histogram of $Z_{16}(\lambda)$.

During the actual experimental process, errors caused by environmental factors were inevitable. Thus, it was necessary to determine the validity of the data. Sixty-five independent repeated measurements were performed with one lens, and the statistical distribution histograms of 36 Zernike terms were obtained. Figure 16 shows the $Z_{16}$ distribution as an example, which is similar to the others. Further, the measurement error follows a normal distribution. Table 7 gives the statistical results for $Z_{7}, Z_{8}, Z_{9}, Z_{16}$, and $Z_{25}$. According to the criterion based on the value of $3 \sigma$, no abnormal data exist in these measurements. Figure 17 shows the difference in the mean wavefront between two adjacent measurements, which is $<\lambda / 50$. The maximum wavefront difference between two adjacent measurements is $<\lambda / 5$. The wavefront data are thus considered stable for perfect imaging. ${ }^{42}$

Table 7 Statistical results for $Z_{7}, Z_{8}, Z_{9}, Z_{16}$, and $Z_{25}$.

\begin{tabular}{lccccc}
\hline \hline Term & $Z_{7}(\lambda)$ & $Z_{8}(\lambda)$ & $Z_{9}(\lambda)$ & $Z_{16}(\lambda)$ & $Z_{25}(\lambda)$ \\
\hline Mean & 0.090318 & 0.051920 & 0.492910 & -0.792187 & -0.165534 \\
Standard deviation & 0.003003 & 0.003429 & 0.001623 & 0.001438 & 0.001336 \\
\hline \hline
\end{tabular}

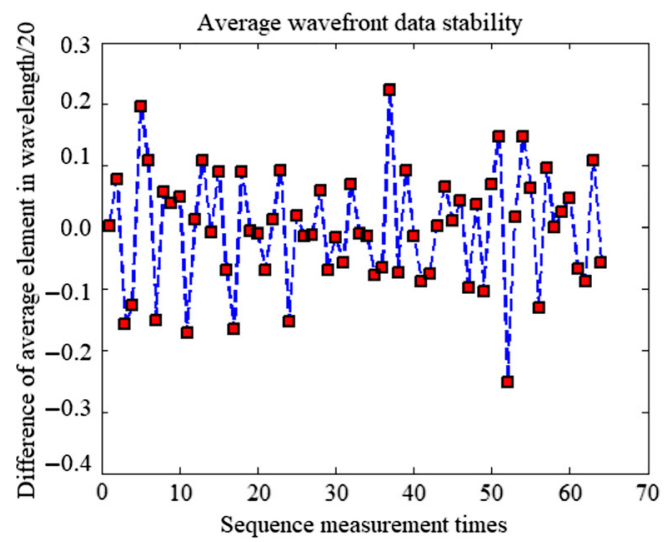

(a)

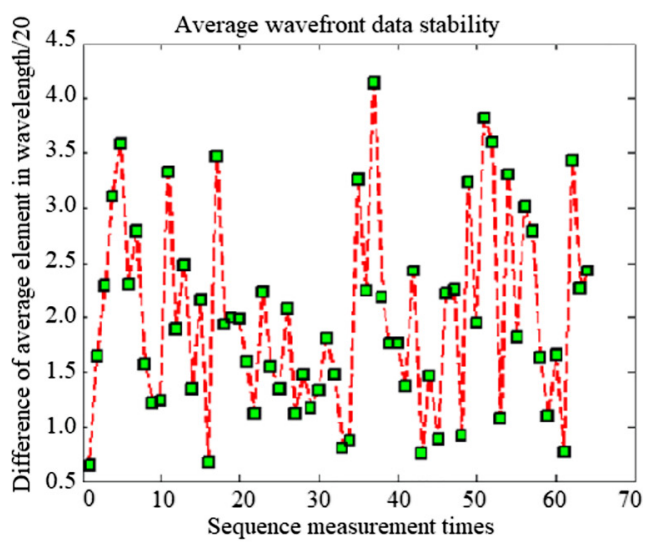

(b)

Fig. 17 Two adjacent wavefront measurements: (a) difference between means; (b) difference between maximum values. 


\section{Acknowledgments}

This research was supported by grants from the Shenzhen Science and Technology Innovation Program (Grant No. JCYJ20170412171011187), the National Natural Science Foundation of China (Grant Nos. 61527826 and 51735002), and the National Key Research and Development Program of China (Grant No. 2017YFC1403602). The authors declare no conflicts of interest.

\section{References}

1. A. Yabe, "Optimal selection of aspheric surfaces in optical design," Opt. Express 13(18), 7233-7242 (2005).

2. M. Heckele and W. K. Schomburg, "Review on micro molding of thermoplastic polymers," J. Micromech. Microeng. 14(3), R1-R14 (2004).

3. V. Piotter et al., "Performance and simulation of thermoplastic micro injection molding," Microsyst. Technol. 8, 387-390 (2002).

4. X. Lu and L. S. Khim, "A statistical experimental study of the injection molding of optical lenses," J.f Mater. Process. Technol. 113(1-3), 189-195 (2001).

5. A. M. Harris and E. C. Lee, "Improving mechanical performance of injection molded PLA by controlling crystallinity," J. Appl. Polym. Sci. 107(4), 2246-2255 (2008).

6. H. Shi, S. Xie, and X. Wang, "A warpage optimization method for injection molding using artificial neural network with parametric sampling evaluation strategy," Int. J. Adv. Manuf. Technol. 65, 343-353 (2013).

7. X. Lan et al., "Optimization of injection molding process parameters and axial surface compensation for producing an aspheric plastic lens with large diameter and center thickness," Appl. Opt. 58(4), 927-934 (2019).

8. F. Z. Fang et al., "Manufacturing and measurement of freeform optics," CIRP J. Manuf. Sci. Technol. 62(2), 823-846 (2013).

9. G. Baer et al., "Calibration of a non-null test interferometer for the measurement of aspheres and free-form surfaces," Opt. Express 22(25), 31200-31211 (2014).

10. I. Fortmeier et al., "Evaluation of absolute form measurements using a tilted-wave interferometer,' Opt. Express 24(4), 3393-3404 (2016).

11. J. C. Wyant, "Computerized interferometric surface measurements," Appl. Opt. 52(1), 1-8 (2013).

12. J. Millerd et al., "Pixelated phase-mask dynamic interferometer," Proc. SPIE 5531, 304-314 (2004).

13. P. J. de Groot, "A review of selected topics in interferometric optical metrology," Rep. Prog. Phys. 32(5), 82 (2019).

14. C. W. Li, B. M. Li, and S. J. Zhang, "Phase retrieval using a modified Shack-Hartmann wavefront sensor with defocus," Appl. Opt. 53(4), 618-624 (2014).

15. R. G. Lane and M. Tallon, "Wave-front reconstruction using a Shack-Hartmann sensor," Appl. Opt. 31(32), 6902-6908 (1992).

16. H. E. Lai and P. J. Wang, "Study of process parameters on optical qualities for injectionmolded plastic lenses," Appl. Opt. 47(12), 2017-2027 (2008).

17. L. Li, T. W. Raasch, and A. Y. Yi, "Simulation and measurement of optical aberrations of injection molded progressive addition lenses," Appl. Opt. 52(24), 6022-6029 (2013).

18. L. Huang et al., "Wavefront measurement of plastic lenses for mobile-phone applications," Adv. Opt. Technol. 5(4), 343-349 (2016).

19. Y. D. Hezaveh, L. P. Levasseur, and P. J. Marshall, "Fast automated analysis of strong gravitational lenses with convolutional neural networks," Nature 548, 555-557 (2017).

20. R. Navarro and E. Moreno-Barriuso, "Laser ray-tracing method for optical testing," Opt. Lett. 24(14), 951-995 (1999).

21. C. Tien, S. Jyu, and H. Yang, "A method for fringe normalization by Zernike polynomial," Opt. Rev. 16, 173-175 (2009).

22. R. J. Bensingh et al., "Injection molding process optimization of a bi-aspheric lens using hybrid artificial neural networks (ANNs) and particle swarm optimization (PSO)," Measurement 134, 359-374 (2019). 
23. W. Michaeli et al., "Geometrical accuracy and optical performance of injection moulded and injection-compression moulded plastic parts," CIRP Ann. Manuf. Technol. 56(1), 545-548 (2007).

24. J. Sasian, "Theory of sixth-order wave aberrations," Appl. Opt. 49(16), 69-95 (2010).

25. R. W. Gray et al., "An analytic expression for the field dependence of Zernike polynomials in rotationally symmetric optical systems," Opt. Express 20(15), 16436-16449 (2012).

26. G. W. Forbes, "Shape specification for axially symmetric optical surfaces," Opt. Express 15(8), 5218-5226 (2007).

27. Z. W. Jiao et al., "Development of internal circulation two-platen IMM for thermoplastic polymer," J. Mater. Process. Technol. 211(6), 1076-1084 (2011).

28. R. N. Dave and R. Krishnapuram, "Robust clustering methods: a unified view," IEEE T. Fuzzy Syst. 5(2), 270-293 (1997).

29. R. Xu and D. C. Wunsch II, "Survey of clustering algorithms," IEEE Trans. Neural Networks 16(3), 645-678 (2005).

30. A. K. Jain, "Data clustering: 50 years beyond K-means," Pattern Recognit. Lett. 31(8), 651-666 (2010).

31. M. Ankerst, M. M. Breunig, H. P. Kriegel, and J. Sander, "OPTICS: ordering points to identify the clustering structure," in Proc. ACM SIGMOD Int. Conf. Manage. Data, pp. 4960 (1999).

32. P. J. Rousseeuw, "Silhouettes: a graphical aid to the interpretation and validation of cluster analysis," J. Computat. Appl. Math. 20, 53-65 (1987).

33. J. C. Bezdek, R. Ehrlich, and W. Full, "FCM: the fuzzy $c$-means clustering algorithm," Comput. Geosci. 10, 191-203 (1984).

34. N. R. Pal and J. C. Bezdek, "On cluster validity for the fuzzy c-means model," IEEE Trans. Fuzzy Syst. 3(3), 370-379 (1995).

35. H. He and E. A. Garcia, "Learning from imbalanced data," IEEE Trans. Knowl. Data Eng. 21(9), 1263-1284 (2009).

36. I. Pillai, G. Fumera, and F. Roli, "F-measure optimisation in multi-label classifiers," in Proc. Int. Conf. Pattern Recognit., pp. 2424-2427 (2012).

37. F. Sebastiani, "Machine learning in automated text categorization," ACM Comput. Surv. 34(1), 1-47 (2002).

38. Y. Yang, "A study of thresholding strategies for text categorization," in Proc. Int. ACM SIGIR Conf. Res. Dev. in Inf. Retrieval, pp. 137-145 (2001).

39. Panasonic Corporation, "UA3P for optical devices: ultrahigh accurate 3-D profilometer," https://industrial.panasonic.com/ (2018).

40. Zygo Corporation, "Compass ${ }^{\mathrm{TM}}$ : micro lens process metrology systems," https://www.zygo .com/ (2019).

41. Trioptics Corporation, "Wavefront sensor: WaveSensor ${ }^{\circledR}, "$ http://trioptics.com/products/ wavemaster-wavefront-measurement-shack-hartmann/ (2019).

42. H. Hopkins, Wave Theory of Aberrations, Oxford University Press, New York (1950).

Xuemin Cheng is an associate professor at the Precision Instrument Department, Tsinghua University, Shenzhen Campus, China. She is the author of more than 80 articles and has developed more than 70 inventions. Her research interests include optical design and engineering, including imaging system design, illumination system design, optical modeling, and simulation. Her current projects include stabilized zoom lens design and application, instrumentation for polarized microscopy, instrumentation for imaging and classification, compressive imaging, and smart imaging through scattering media. She is a member of SPIE.

Lei Yan is currently pursuing his $\mathrm{PhD}$ in instrumentation and device technology with Beijing Institute of Technology, China. His research mostly involves the completion of a project on a zooming imaging, and aberration identification and compensation instrument, used for in situ monitoring and intelligent imaging.

Lingcheng Liu is currently pursuing the master's degree in instrumentation and device technology with Tsinghua University, China. His research mostly involves the completion of a 
project on a lens inspection and aberration identification device, used for in situ monitoring and intelligent identification of lens quality.

Jie Cao received his $\mathrm{PhD}$ in school of optics and photonics from Beijing Institute of Technology, China, in 2015. Then, he completed his postdoc at the National University of Singapore. Now, he is an associate research fellow in Beijing Institute of Technology, and interested fields include bio-inspired vision and 3D imaging.

Yu Jen Lin received his master's degree in school of optical sciences from National Central University, China, Taiwan, in 2001. Now, he is a director of test engineering Department of Genius Electronic Optical (Xia Men) Co. Ltd. and interested fields include freeform metrology, mobile phone lens MTF test.

Qun Hao received her PhD in optical instruments from Tsinghua University, China, in 1997. She became a full professor in 2003 and currently serves as the dean of school of optics and photonics in Beijing Institute of Technology. She is the chairman of Optics Council in China Ordnance Society, the director of China Instrument and Control Society and director of Chinese Society for Measurement. 